\title{
Implementación de la fase uno del proyecto "Educación financiera gamificada como estrategia didáctica para el desarrollo de estilos de vida sostenibles"
}

\author{
Martha Liliana Torres Barretoa ${ }^{a}$ Karen Rocío Plata Gómez ${ }^{b}$, Silvia Nathalia Núñez \\ Rueda ${ }^{c}$ \\ ${ }^{a}$ Universidad Industrial de Santander (Bucaramanga, Colombia); mltorres@uis.edu.co buniversidad \\ Industrial de Santander (Bucaramanga, Colombia); karengomez920@gmail.com ${ }^{\mathrm{c}}$ Universidad \\ Industrial de Santander (Bucaramanga, Colombia); silvia.nunez@est.uexternado.edu.co.
}

\begin{abstract}
Resumen
Los productos financieros actuales ofrecen una asequibilidad sin precedentes, lo cual facilita nuevas oportunidades para la inclusión financiera de los jóvenes. Sin embargo, esto conlleva numerosos riesgos asociados a su bajo conocimiento acerca de los beneficios y desventajas de los diferentes productos financieros. Esto se asocia, entre otras causas, a la baja implementación de programas de Educación Económica y Financiera EEF- en las instituciones educativas. Para el caso de Colombia, los resultados de las mediciones de capacidades económicas y financieras son poco favorables, pues evidencian bajos niveles de conocimiento en finanzas personales por parte de los estudiantes de educación básica y media del país (OECD, 2014).

Este trabajo presenta los primeros hallazgos obtenidos tras el diagnóstico de las capacidades económicas y financieras de 614 estudiantes pertenecientes a 5 instituciones educativas de la región de Santander, en Colombia, mediante la aplicación de un instrumento validado por la OECD. Los resultados preliminares muestran que los hallazgos encontrados, concuerdan con los presentados en la literatura, donde se evidencia que efectivamente los comportamientos financieros (es decir, la habilidad, la actitud y el conocimiento) de los estudiantes se ven afectados positivamente por la implementación de la educación financiera impartida a través del currículo escolar.
\end{abstract}

Palabras clave: Educación económica y financiera, conocimientos financieros, enseñanza, secundaria y gamificación. 


\section{Introducción}

En la actualidad la educación económica y financiera - EEF- recibe una atención considerable entre los investigadores, dado que es uno de los principales factores que contribuyen al crecimiento económico y al desarrollo de las naciones (Liu et al., 2019). Sin embargo, se reconoce también que el comportamiento financiero irresponsable y las habilidades financieras deficientes son las principales causas de la aparición de deudas, no solo entre los adultos, sino también entre los jóvenes, quienes se identifican como un grupo particularmente vulnerable (Amagir et al., 2018). Además, como la mayoría de las transacciones en el mundo contemporáneo son monetarias, los productos financieros se están volviendo cada vez más complejos, por lo que adquirir habilidades de gestión financiera es más importante. Por ello, es necesario empoderar a los jóvenes desde edades tempranas para que sean "ciudadanos responsables en los ámbitos económico y financiero, de modo que incidan en la mejora de las condiciones sociales, económicas, políticas, administrativas y culturales" (MEN \& Asobancaria, 2014).

En este sentido, el método más poderoso para mejorar la EEF de los jóvenes es aumentar la cobertura de las finanzas personales en el currículo escolar, lo cual debe promoverse desde el gobierno hacia las instituciones educativas (Jang et al., 2014). Independiente del enfoque que decidan emplear, estos programas deben ser diseñados para satisfacer las necesidades y el nivel de conocimientos financieros de la población, generando así cambios en su bienestar financiero (Asobancaria, 2016; OECD, 2005).

En Colombia los resultados en pruebas estandarizadas de los estudiantes en temas de conocimientos económicos y financieros han sido desalentadores. Entre los países evaluados en la prueba PISA 2018, el país obtuvo el puesto 58 de 79 países que se presentaron (OECD, 2020). Dicha situación demuestra que es necesario educar económica y financieramente a la población del país. Sin embargo, en la actualidad el sistema educativo requiere un cambio de enfoque en el componente metodológico y didáctico en la formación de los estudiantes en el área de EEF. Ante esta necesidad surge la gamificación como una estrategia para desarrollar el potencial del alumnado por medio de la dinámica de juego, con el propósito de despertar en el estudiante el interés por aprender (Torres-Barreto, 2018).

Teniendo en cuenta lo anterior, se formula el proyecto "Educación financiera gamificada como estrategia didáctica para el desarrollo de estilos de vida sostenibles", con el objetivo de aportar al proceso pedagógico de EEF de los jóvenes colombianos, mediante el uso de una herramienta gamificada, acorde a la realidad y necesidades de esta población. Para el desarrollo del proyecto, se ha planeado la ejecución de cuatro fases: Fase 1. Diagnóstico, Fase 2. Diseño de la herramienta gamificada, Fase 3. Alcances y limitaciones y Fase 4. 
Análisis y divulgación. Entre enero y principios de mayo de 2020, se ejecutó gran parte de la Fase 1, en la cual se identificaron preliminarmente las capacidades económicas y financieras de los estudiantes de las instituciones participantes del proyecto. Por tanto, a continuación se presentan los resultados preliminares obtenidos tras la implementación de la Fase 1 del proyecto.

\section{Revisión de tendencias}

Dadas las políticas y requisitos educativos para la educación en el área de finanzas personales, diversos autores coinciden en que es necesario atender esta necesidad e incorporar al currículo escolar programas que permitan educar económica y financieramente a los estudiantes, iniciando desde la escuela primaria, repitiendo en la escuela secundaria y continuando en la universidad (Amagir et al., 2018).

Bajo este escenario, a nivel internacional en países como Canadá, Estados Unidos y España la EEF es obligatoria en casi todos los grados del colegio, lo cual han logrado a través de la implementación y articulación de sus planes de estudio y estrategias nacionales de alfabetización (Asobancaria, 2018a). Por un lado, Canadá cuenta con la Estrategia Nacional para la Educación Financiera "Cuenta conmigo", que moviliza los sectores público, privado y sin fines de lucro y busca su compromiso para fortalecer la educación financiera de los canadienses y capacitarlos correctamente para administrar su dinero y deudas, planear y ahorrar para su futuro y prevenir y protegerse contra el fraude y el abuso financiero (Financial Consumer Agency of Canada, 2019).

Por otra parte, Estados Unidos cuenta con los Estándares Nacionales de Educación en Finanzas Personales K-12, de la Coalición para la alfabetización financiera personal Jump\$tart, (2007). Dichos estándares delinean el conocimiento y la capacidad en finanzas personales que los jóvenes deben adquirir a lo largo de los años, desde jardín de infantes hasta $12^{\circ}$ grado (K-12) de escolaridad para emerger como consumidores adultos independientes, preparados para tomar decisiones. Mientas que, España ha implementado un Plan de Educación Financiera en Enseñanza Secundaria Obligatoria emitido por el Ministerio de Educación, el Banco de España y la Comisión Nacional del Mercado de Valores, con el objetivo de potenciar los conocimientos, destrezas y habilidades de corte económico que permitan al alumnado comprender la importancia de conceptos clave como el ahorro, el presupuesto, los gastos, los ingresos, etc (CNMV \& Banco de España, 2018).

Del mismo modo, algunas entidades expertas y sin ánimo de lucro a nivel global, como la Microfinances Oportunities y la Fundación de las Cajas de Ahorro de Alemania (Sparkassenstiftung), se han centrado en impulsar la educación y el sector financiero local 
de países en vías de desarrollo, principalmente de América Latina y el Caribe. Estas entidades trabajan diseñando e implementando las metodologías y planes de estudio de EEF que han logrado desarrollar, adaptándolas a las necesidades y el contexto local de cada país, con el objetivo de incrementar el nivel de conocimientos financieros de la población y promover el fortalecimiento de la inclusión financiera para reducir la pobreza (Asobancaria, 2015, 2018b).

En cuanto al impacto de su implementación en Colombia, a través del uso del "Financiometro", que es un sistema que integra conocimientos y actitudes ${ }^{1}$, se ha logrado medir los resultados obtenidos tras su implementación, evidenciando mejoras en el score crediticio de las personas sometidas a estos programas en los años posteriores (Asobancaria, 2018b).

\section{Metodología}

Para el desarrollo del proyecto se ha planeado la ejecución de cuatro fases: la Fase 1. Diagnóstico: Análisis de la situación y definición del problema; Fase 2. Diseño de la herramienta: Desarrollo de soluciones; Fase 3. Alcances y limitaciones: Implementación y Validación y Fase 4. Análisis-Divulgación: Producción de documentación y principios de diseño. Para mayo de 2020, se ejecutó gran parte de la Fase 1 que comprendió el diseño del cuestionario de capacidades económicas y financieras, la recolección de la información con los estudiantes de las instituciones participantes, el procesamiento de los datos, y por último el análisis de la información recolectada.

Diseño del cuestionario de capacidades financieras. Para medir las capacidades económicas y financieras de los estudiantes participantes del estudio, se usó como base el cuestionario OECD/INFE Toolkit for measuring financial literacy and financial inclusion (OCDE, 2020). Este instrumento se escogió dado que proporciona información sobre la planeación y administración de las finanzas, uso de productos financieros, conductas y actitudes relacionadas con el dinero y conocimientos financieros de quienes lo desarrollan.

En la preparación y ajuste del cuestionario se tuvo en cuenta, además, otros instrumentos usados en algunas mediciones de capacidades financieras para Colombia (Corporación Andina de Fomento, 2015) con el fin de realizar la traducción y adaptación de lenguaje. Una vez surtido este proceso, se determinó necesario por parte del equipo de proyecto ajustar algunos ítems del mismo, y realizar una adaptación de algunas preguntas.

\footnotetext{
${ }^{1}$ Estas últimas medidas por el comportamiento crediticio de los beneficiarios de los programas que aceptan ser parte de esta medición.
} 
Una vez realizada esta revisión, se estructuraron 41 preguntas, distribuidas en 10 componentes. Estos corresponden a los siguientes: i) Características personales y del hogar; ii) Información de contexto; iii) Planificación de finanzas y gestión de finanzas; iv) Ahorro activo y choques financieros; v) Metas financieras; vi) Planificación financiera a largo plazo; vii) Gastos mensuales; viii) Actitudes y comportamiento; ix) Conocimiento financiero; y x) Conocimiento de productos financieros.

Recolección y tratamiento de la información. Antes de la aplicación del cuestionario, en el mes de marzo se realizó una prueba piloto del instrumento traducido y ajustado al español con un grupo de 220 estudiantes de undécimo grado de una de las instituciones participantes. Posterior a esta prueba, se ajustaron algunas preguntas, opciones de respuesta, términos y número de preguntas por cada componente del cuestionario. Entre el mes de abril y parte del mes de mayo, los estudiantes participantes, con el permiso previo de su tutor, diligenciaron la versión final del cuestionario a través de la herramienta Google Forms. En total se obtuvieron 614 respuestas. Para el tratamiento de los datos, el equipo de proyecto implementó el uso del software de analítica Tableau, el cual se referencia como una potente herramienta de visualización de información, además que brinda la facilidad para integrar diferentes tipos de datos.

Población participante. Para el estudio se seleccionaron estudiantes de educación media ${ }^{2}$ de 5 instituciones del departamento de Santander (Colombia). Las instituciones educativas se seleccionaron teniendo en cuenta las alianzas y cercanía institucional con la Universidad Industrial de Santander (UIS), institución de los investigadores asociados al proyecto.

\section{Resultados preliminares}

A continuación, se presentan los resultados preliminares más relevantes del procesamiento y análisis de algunos de los componentes del cuestionario diligenciado por los participantes.

Características personales y del hogar. En la distribución por género se encuentra que el $46,4 \%$ de los estudiantes son hombres, un $53 \%$ mujeres y un $0,49 \%$ prefirió no referirlo. En cuanto a la distribución por edad, la mayor cantidad se ubicó en los 16 años con un $56,2 \%$. Referente a su participación por estrato socioeconómico ${ }^{3}$, la mayoría se ubican en el

\footnotetext{
2 El sistema educativo colombiano lo conforman: la educación inicial, la educación preescolar, la educación básica (primaria cinco grados y secundaria cuatro grados), la educación media (dos grados y culmina con el título de bachiller), y la educación superior.

3 La estratificación socioeconómica es una clasificación en estratos de los inmuebles residenciales que deben recibir servicios públicos. De éstos, los estratos 1, 2 y 3 corresponden a estratos bajos que albergan a los usuarios con menores recursos; los estratos 5 y 6 corresponden a estratos altos que albergan a los usuarios con mayores recursos económicos. El estrato 4 no es beneficiario de subsidios, ni debe pagar sobrecostos.
} 
estrato tres con un 50,3\% del total. Además, se tiene una participación importante de estudiantes del estrato dos $(27,8 \%)$ y de estrato uno $(8,2 \%)$.

Información de contexto. A la pregunta ¿Cómo calificaría su conocimiento general sobre asuntos financieros en comparación con otros jóvenes en Colombia? La mayoría $(63,6 \%)$ manifestó que sus conocimientos se encuentran sobre el promedio. Sin embargo, es relevante que un $14,6 \%$ consideran que sus conocimientos en el tema son "bajos" y un $9,6 \%$ los consideran como "muy bajos".

Planificación de finanzas y gestión de finanzas. En este componente, se cuestionó sobre el manejo de dinero propio y la autonomía en el manejo de estos recursos. Al respecto, se observa que un $79,6 \%$ manifiesta tomar decisiones sobre su dinero propio. A la par con esta pregunta, se cuestionó a los estudiantes sobre qué acciones relacionadas con estos recursos o los de su hogar realiza, se observa que un $41,6 \%$ hace un plan para administrar sus ingresos y gastos, un $43,7 \%$ toma nota de sus gastos, un 52,8\% mantiene el dinero para los gastos fijos separado del dinero que gasta día a día y un 6,2\% usa una aplicación bancaria o una herramienta de administración de dinero para realizar un seguimiento de sus gastos.

Ahorro activo y choques financieros. En este componente se preguntó a los estudiantes en los últimos 12 meses de qué maneras han estado ahorrando dinero. De las opciones presentadas la gran mayoría manifestaron que ahorraron dinero en efectivo en una alcancía o en su billetera. Adicionalmente, se cuestionó a los estudiantes por su capacidad para enfrentar un gasto importante sin necesidad de pedir prestado a sus familiares o amigos. Al respecto, un $42,2 \%$ señaló que tal vez podría hacerlo, un 32,4\% señaló que podría hacerlo y un $11,4 \%$ afirma poder hacerlo sin tener que pedir prestado dinero.

Metas financieras. Se encuentra que un $88,5 \%$ de los estudiantes manifiestan tener algún tipo de meta financiera. A quienes contestaron afirmativamente, se les cuestionó sobre ¿Cuál es esta meta financiera? A lo anterior, un 34,1\% mencionó que pagar sus estudios universitarios es la meta financiera principal. En otras respuestas, se observa que un 11,4\% planea invertir en una casa propia, un $8,3 \%$ comparar un celular y un $4.5 \%$ crear una empresa.

Conocimiento financiero. Para conocer el nivel de conocimiento económico y financiero de los participantes, se realizaron cinco preguntas en las cuales los estudiantes tenían que aplicar algunos conocimientos matemáticos básicos para resolverlos. Es relevante que aquellas preguntas que requerían operaciones matemáticas más sencillas para llegar a la respuesta correcta fueron las que tuvieron porcentajes de acierto más altos, pero aquellas que requerían más de una operación matemática, y además, el conocimiento de términos como inflación, tasa de interés o cuenta de ahorros fueron las de más bajo nivel de acierto. 
Conocimiento de productos financieros. En lo referente a los productos financieros de deuda se observó que los más conocidos por los estudiantes son: tarjeta de crédito y crédito de vivienda. Referente a los productos financieros de inversión se encuentra que los productos que más conocen son: cuenta de ahorro e inversiones en la bolsa.

\section{Conclusiones}

Con más frecuencia las personas se sobreendeudan y enfrentan dificultades para administrar sus finanzas personales. Tal situación podría conducir a problemas personales (dificultades financieras), agravamiento de la estabilidad financiera, con consecuencias adversas sobre el crecimiento económico. En este sentido, tal como se expuso al inicio del documento es necesario la creación de herramientas que permitan a los estudiantes desde temprana edad construir habilidades económicas y financieras que les permitan tener un comportamiento responsable de sus finanzas personales.

De acuerdo con los resultados preliminares presentados, es importante resaltar que los estudiantes tienen entre sus productos financieros más conocidos tarjetas de crédito y crédito de vivienda, instrumentos de deuda que requieren un conocimiento previo para su manejo eficiente, pues tal como lo mencionan Lusardi \& Mitchell, (2014) las nuevas oportunidades de inclusión financiera ponen a disponibilidad de los más jóvenes la adquisición de diferentes productos y servicios financieros, facilitando o dando acceso relativamente libre al crédito, por lo cual se hace necesario ofrecer a los jóvenes las herramientas necesarias para su administración.

Adicionalmente, en las respuestas obtenidas en el componente de conocimientos financieros, se evidencia un desconocimiento de términos como: inflación y tasa de interés, los cuales resultan fundamentales para comprender problemas económicos y financieros básicos que cualquier persona debe entender para un manejo eficiente de sus finanzas personales. Esto concuerda con lo expuesto por Walstad et al., (2017), quienes a través de su estudio manifiestan que una de las principales razones para fomentar el desarrollo de habilidades económicas y financieras desde las diferentes etapas escolares, es proporcionar una base para desarrollar la comprensión de los conceptos financieros básicos para resolver problemas financieros que los estudiantes pueden experimentar más adelante en la vida. En general, los hallazgos encontrados, concuerdan con los presentados en la literatura, donde se evidencia que efectivamente los comportamientos financieros (es decir, la habilidad, la actitud y el conocimiento) de los estudiantes se ven afectados positivamente por la implementación de la educación financiera impartida a través del currículo escolar (Cordero \& Pedraja, 2019). 
Ahora bien, los resultados anteriores serán la base para la construcción de una herramienta gamificada que permita el desarrollo de habilidades económicas y financieras, que suplan las necesidades de los jóvenes del departamento de Santander (Colombia) en temas de EEF, y que además, puedan apoyar a las instituciones educativas en la formación en esta área. Adicionalmente, se espera construir la herramienta gamificada en el segundo semestre de 2020, teniendo en cuenta lo enunciado en la literatura académica y lo abordado en otros programas educativos internacionales.

Asimismo, se espera que en años posteriores a partir de los resultados obtenidos en este proyecto, se continúe con el diseño de herramientas gamificadas que contribuyan en el mejoramiento de la educación económica y financiera de los jóvenes de secundaria en Colombia.

\section{Referencias}

Amagir, A., Groot, W., Maassen van den Brink, H., \& Wilschut, A. (2018). A review of financialliteracy education programs for children and adolescents. Citizenship, Social and Economics Education, 17(1), 56-80. https://doi.org/10.1177/2047173417719555

Asobancaria. (2015). Educación Económica y Financiera: Guía de orientación para el diseño y puesta en marcha de políticas territoriales Asociación Bancaria y de Entidades Financieras de Colombia. Bogotá.

Asobancaria. (2016). Programas de Educación Financiera en Latinoamérica : un énfasis en el caso colombiano. Semana Económica 2016, 1-13.

Asobancaria. (2018a). ¿Por qué debemos implementar el programa de Educación Financiera en los colegios de Colombia?

Asobancaria. (2018b). Victorias y retos de la educación financiera en 2017.

Corporación Andina de Fomento. (2015). Encuesta de Medición de Capacidades Financieras en los Países Andinos. Informe para Colombia 2014. Retirado de: https://scioteca.caf.com/bitstream/handle/123456789/743/ENCUESTA DE MEDICION DE LAS CAPACIDADES FINANCIERAS EN LOS PAÍESE ANDINOS COLOMBIA.pdf? sequence $=5$ \&isAllowed $=\mathrm{y}$

CNMV, \& Banco de España. (2018). Plan de Educación Financiera 2018-2021. Madrid.

Cordero, J. M., \& Pedraja, F. (2019). The effect of financial education training on the financial literacy of Spanish students in PISA. Applied Economics, 51(16), 1679-1693. https://doi.org/10.1080/00036846.2018.1528336

DANE (2020). Estratificación socioeconómica para servicios públicos domiciliarios. Disponible en: https://www.dane.gov.co/index.php/servicios-al-ciudadano/servicios-informacion/estratificacionsocioeconomica 
Financial Consumer Agency of Canada. (2019). Implementing the National Strategy for Financial Literacy - Count me in, Canada. Progress report 2015-2019.

Jang, K., Hahn, J., \& Park, H. J. (2014). Comparison of financial literacy between Korean and U.S. high school students. International Review of Economics Education, 16(PA), 22-38. https://doi.org/10.1016/j.iree.2014.07.003

Jump\$tart. (2007). National standards in $\mathrm{K}-12$ personal finance education. Retrieved from https://www.jumpstart.org/wp-content/uploads/2018/01/2017_NationalStandardsBook.pdf

Liu, C. L., Chang, D. F., \& Chuang, C. M. (2019). Investigation of the financial literacy among high school students. ICIC Express Letters, Part B: Applications, 10(5), 387-393. https://doi.org/10.24507/icicelb.10.05.387

Lusardi, A., \& Mitchell, O. (2014). The economic importance of financial literacy: Theory and evidence. Journal of Economic Literature, 52(1), 5-44.

MEN, \& Asobancaria. (2014). Mi vida, mi plan, mi futuro. Orientaciones pedagógicas para la educación económica y financiera. Bogotá.

OECD. (2005). Improving financial literacy: analysis of issues and policies.

OCDE (2018). OCDE/INFE: Toolkit for Measuring Financial Literacy and Financial Inclusion. Recuperado de: http://www.oecd.org/daf/fin/financial-education/2018-INFEFinLit-MeasurementToolkit.pdf

OECD (2020), PISA 2018 Results (Volume IV): Are Students Smart about Money?, PISA, OECD Publishing, Paris, https://doi.org/10.1787/48ebd1ba-en.

Torres-Barreto, Martha Liliana. (2018). Herramienta didáctica motivacional basada en gamificación y apoyada en TIC para adquirir y aplicar competencias transversales en estudiantes de ingeniería: MOTIVATIC. ffhal-02166319

Walstad, W., Urban, C., J. Asarta, C., Breitbach, E., Bosshardt, W., Heath, J., ... Xiao, J. J. (2017). Perspectives on evaluation in financial education: Landscape, issues, and studies. Journal of Economic Education, 48(2), 93-112. https://doi.org/10.1080/00220485.2017.1285738 